\title{
Auswirkungen des Klimawandels auf die Verfügbarkeit von Kältewirkung (Chilling) für Obstgehölze in Deutschland
}

\author{
Eike Luedeling $\cdot$ Michael Blanke $\cdot$ Jens Gebauer
}

Eingegangen: 6 April 2009 / Angenommen: 16 Mai 2009 / Online veröffentlicht: 22 Juli 2009

(C) Die Autoren 2009

Zusammenfassung Um die Auswirkungen des Klimawandels auf den deutschen Obstbau zu quantifizieren, befasst sich diese Arbeit mit langzeitlichen Trends in der Verfügbarkeit von Kältewirkung (Chilling) für Obstgehölze. Die Berechnungen erfolgten nach dem Kältestunden-Modell und dem Kälteportionen-Modell (Dynamisches Modell).

Aus täglichen Aufzeichnungen von 43 Wetterstationen wurden mittels idealisierter täglicher Temperaturkurven stündliche Temperaturen während des 20. und späten 19. Jahrhunderts abgeschätzt und daraus die Kältewirkung für jedes Jahr errechnet. Neben zeitlichen Trends konnten daraus durch räumliche Interpolation flächendeckende Karten des zu erwartenden Chillings um das Jahr 2010 und der Veränderungen seit 1950 errechnet werden.

Insgesamt bewegte sich die Kältewirkung zwischen etwa 1700 und 3000 Kältestunden bzw. zwischen 125 und 150 Kälteportionen, wobei das meiste Chilling in den eher milden nördlichen Regionen anfiel. Deutschlandweit scheint es dabei bisher keine signifikanten zeitlichen Trends zu geben.

Wie stark sich die verschiedenen Regionen Deutschlands hinsichtlich der Kältewirkung unterscheiden, hängt von

E. Luedeling

University of California Davis, Department of Plant Sciences, One Shields Ave, Davis 95616, CA, USA

E-Mail: eluedeling@ucdavis.edu

M. Blanke

Universität Bonn, INRES - Gartenbauwissenschaften,

Auf dem Hügel 6, 53121 Bonn, Deutschland

E-Mail: ulp304@uni-bonn.de

J. Gebauer (®)

Universität Kassel, Ökologischer Pflanzenbau

und Agrarökosystemforschung in den Tropen und Subtropen,

Steinstr. 19, 37213 Witzenhausen, Deutschland

E-Mail: tropcrops@uni-kassel.de der Wahl des Kältemodells ab. Während das KältestundenModell für die Regionen um Dresden, Leipzig und den Bodensee starke Verluste zeigte, ergab die Auswertung nach dem Kälteportionen-Modell keine derartigen Muster.

Stärker als durch abnehmendes Chilling könnten Obstbauern in Deutschland durch zunehmende Wärme im Winter Probleme bekommen. In einem sehr warmen Winter (z. B. im Rekordwinter 2006/07) können Obstgehölze, die schon früh ihr Kältebedürfnis erfüllt haben, frühzeitig zur Blüte kommen. Das führt zu einem erhöhten Risiko von Frostschäden und die Gleichmäßigkeit der Blüte kann beeinträchtigt werden.

Schlüsselwörter Chilling · Kälteportionen · Kältereiz · Kältestunden $\cdot$ Kältewirkung $\cdot$ Klimawandel

\section{Climate change effects on winter chill for fruit crops in Germany}

Abstract To quantify the effects of climate change on fruit production in Germany, this study aimed at determining long-term trends in winter chill, as calculated with the Chilling Hours and Dynamic Models (Chill Portions). An idealized daily temperature curve was used to convert daily temperature records from 43 weather stations, taken throughout the twentieth and late nineteenth centuries, into an hourly dataset, which was then converted to units of winter chill. Besides exposing temporal trends in winter chill, the data could be spatially interpolated, yielding contiguous maps of typical winter chill in Germany around 2010, as well as chilling losses since 1950.

Throughout Germany, winter chill varied between 1700 and 3000 Chilling Hours or 125 and 150 Chill Portions. The areas of highest winter chill were located in the northern 
parts of the country. For the whole of Germany, there were no significant temporal trends.

The extent of interregional variation in winter chill depended on the chilling model used. While the Chilling Hours Model showed strong declines in winter chill for the areas around Dresden and Leipzig, as well as for the Lake Constance region, the Dynamic Model did not detect such dramatic changes.

More than a decline in winter chill, increased heat during the winter months might become a challenge to German fruit growers. As already experienced during the extraordinarily warm winter of 2006/07, warm temperatures during the winter can cause fruit trees that fulfill their chilling requirements relatively early to bloom prematurely. This can then lead to elevated risk of frost damage and hamper the homogeneity of flowering.

Keywords Chill portions · Chilling hours · Climate change · Winter chill

\section{Einleitung}

Quantifizierung der Kältewirkung

Eine grundlegende Voraussetzung für den erfolgreichen Anbau vieler Obst- und Nussbaumarten ist die Erfüllung des winterlichen Kältebedürfnisses (Saure 1985; Vegis 1961; Westwood 1993). Dieses Bedürfnis muss jeden Winter erfüllt werden, damit die Obstgehölze vollständig aus der Winterruhe erwachen und gleichmäßig blühen. Hierbei wird generell angenommen, dass der Blühzeitpunkt durch das sequenzielle Erfüllen kulturspezifischer Kälteund Wärmebedürfnisse bestimmt wird (Abb. 1). Fehlende Kältewirkung kann bei den Obstgewächsen zu unregelmäßi-

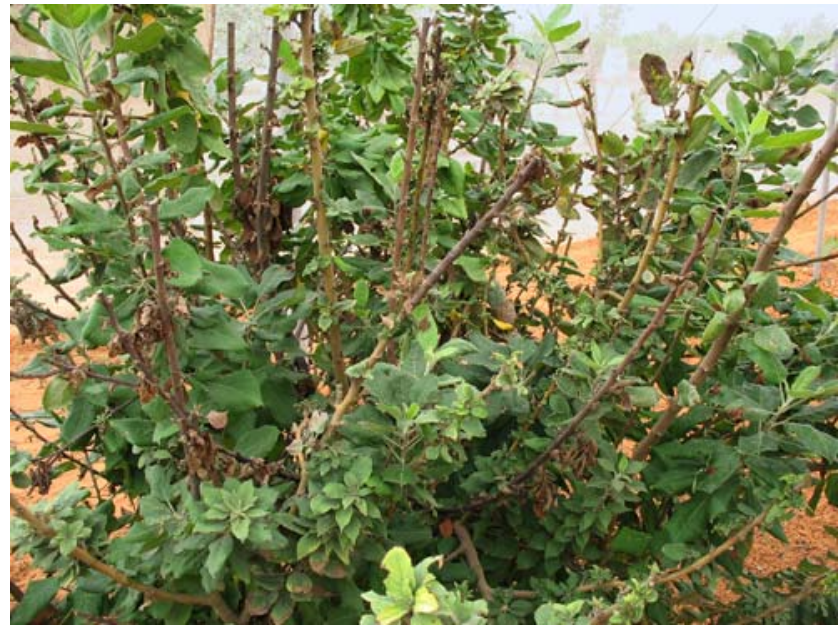

Abb. 2 Auswirkungen der Nichterfüllung des Kältebedürfnisses bei einem Apfelbaum in der Versuchsstation des ICRISAT im Niger

gem Blühablauf, verzögertem vegetativen Wachstum und letztlich zum Vergreisen der Gehölze führen (Abb. 2).

Die Auswirkungen niedriger Temperaturen auf die Dormanzbrechung werden schon seit mehr als zwei Jahrhunderten wissenschaftlich untersucht (Knight 1801), was zur Entwicklung einer Vielzahl von konzeptionellen Erklärungsversuchen geführt hat. Da die meisten dieser Theorien auf sehr begrenzten Datengrundlagen basieren und viele keine Erklärung für experimentelle Ergebnisse aus den letzten Jahrzehnten und Beobachtungen in verschiedenen Klimazonen liefern, verzichten wir hier auf eine erschöpfende Diskussion. Es sei aber auf zwei umfangreiche Literaturstudien zu diesem Thema verwiesen (Saure 1985; Vegis 1961).

In Deutschland war das Thema Kältewirkung lange von untergeordneter Bedeutung, da die relativ langen und kalten
Abb. 1 Konzeptuelle Darstellung der Kälte- und Wärmeakkumulation während der Winterruhe (sequenzielles Erfüllen des Kälte- und Wärmebedürfnisses bestimmt den Blühzeitpunkt)

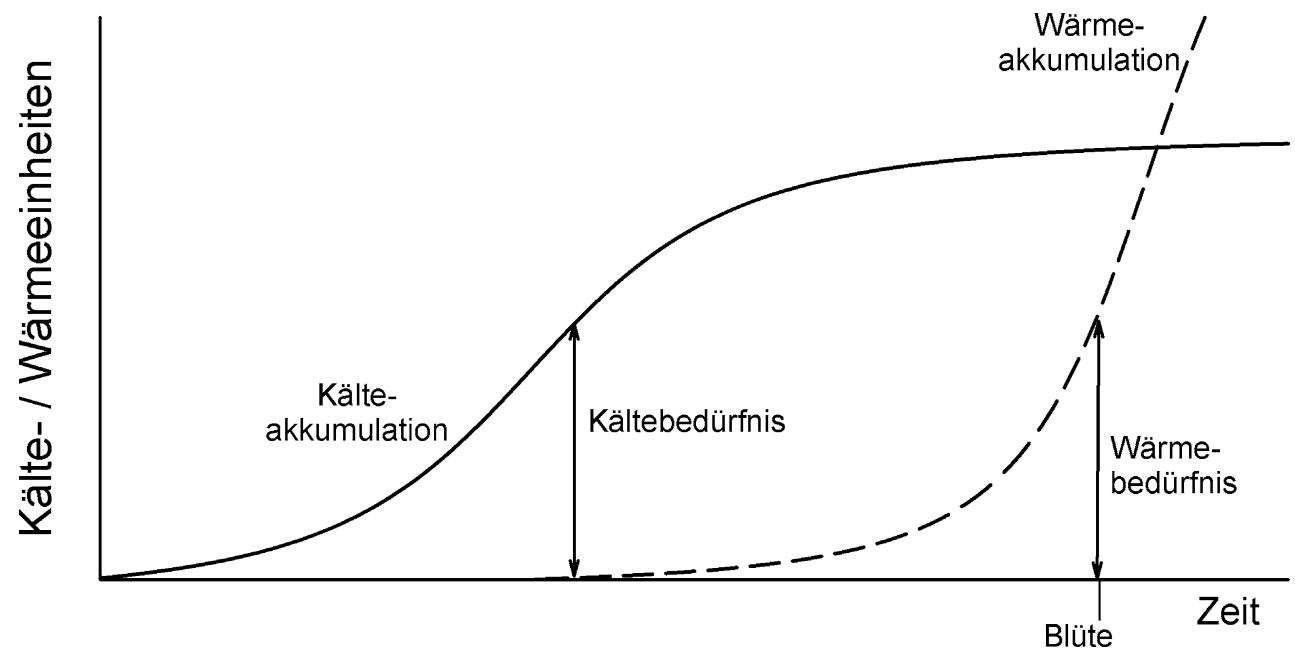


Winter jedes Jahr das Kältebedürfnis unserer Obstgehölze ausreichend erfüllten. Zudem werden hierzulande größtenteils Obstarten und -sorten angebaut, die seit Jahrhunderten in den gemäßigten Breiten beheimatet sind. In anderen Anbaugebieten der Welt ist die Erfüllung des Kältebedürfnisses weit weniger garantiert, und erheblicher Forschungsaufwand wurde betrieben, um Kältewirkung (engl. Chilling, auch im Deutschen häufig verwendet) und Kältebedürfnisse zu quantifizieren. Insbesondere in subtropischen Regionen wie Kalifornien und Israel war diese Forschung die Voraussetzung für den erfolgreichen Anbau von Obst- und Nussarten der gemäßigten Breiten. Der mathematischen Modellierung des Chillings kam hierbei stets besondere Bedeutung zu (Müller u. Braun 2008), was zur Entwicklung einer Vielzahl von Chilling-Modellen geführt hat (Tabelle 1). Generell lässt sich ein Zusammenhang zwischen der Ausgefeiltheit der mathematischen Modelle und der Genauigkeit der Quantifizierung der Kältewirkung feststellen.

Das erste brauchbare Modell ist das in den USA entwickelte Kältestunden-Modell, das Kältewirkung in Kältestunden (engl. Chilling Hours) quantifiziert (Bennett 1949; Weinberger 1950). In Kalifornien erlaubt das Klima generell den Anbau vieler Gehölzarten aus den gemäßig- ten und kühlen Klimaten, erfordert aber die Selektion geeigneter Pfirsich-, Pflaumen-, Walnuss- und Aprikosensorten unter Berücksichtigung der lokal verfügbaren Kältewirkung. Im Kältestunden-Modell wird Chilling durch die Anzahl der Stunden mit Temperaturen zwischen 0 und $7,2^{\circ} \mathrm{C}\left(45^{\circ}\right.$ Fahrenheit) ausgedrückt. Nach Westwood (1993) benötigen Apfelbäume mit $800-1700 \mathrm{~h}$ relativ viele, Süßkirschbäume mit 550-1300 h etwas weniger und Quittenbäume mit 100-500 h relativ wenige Kältestunden. In Kalifornien und vielen anderen Obstanbaugebieten war dieses Modell lange Zeit hinreichend, und auch in Deutschland wird meist mit diesem Modell gearbeitet.

Im Gegensatz dazu wurde in kontinentalen Klimaten mit relativ großen täglichen Temperaturschwankungen festgestellt, dass warme Wintertemperaturen negative Auswirkungen auf die Akkumulation von Chilling haben, und dass sich verschiedene Temperaturbereiche unterschiedlich stark auf die Kältewirkung auswirken. Aus diesen Erkenntnissen entstand das Utah-Modell (Richardson et al. 1974), das in einigen Anbauregionen teilweise in leicht veränderten Versionen (z. B. als North-Carolina-Modell; Shaltout u. Unrath 1983) bis heute Verwendung findet. Obwohl das Utah-Modell inzwischen weiter verbessert

Tabelle 1 Übersicht und Vergleich ausgewählter Kältewirkungs-Modelle hinsichtlich in Experimenten ermittelter Merkmale des ChillingAkkumulationsprozesses

\begin{tabular}{llll}
\hline & & & \\
& & &
\end{tabular}

+ bedeutet, dass das entsprechende Merkmal im betreffenden Modell berücksichtigt wurde, - bedeutet, dass es nicht berücksichtigt wurde, \pm deutet an, dass beides vorkommt.

Die verglichenen Merkmale sind der Temperaturschritt $(\mathrm{h}=$ stündlich, $\mathrm{d}=$ täglich, $\mathrm{m}=$ monatlich), die differenzielle Gewichtung verschiedener Temperaturbereiche, die kontinuierliche (im Gegensatz zu schrittweiser) Gewichtung der Temperaturbereiche, die Aufhebung vorangegangener Kältewirkung durch hohe Temperaturen, die zeitliche Begrenzung dieser Aufhebung, die Verstärkung des Chilling durch nachfolgende moderate Temperaturen sowie die zweiphasige Ansammlung der Kältewirkung.

Nicht in allen Fällen handelt es sich bei den angegebenen Autoren um die Entwickler der Modelle. 
wurde (Anderson u. Seeley 1992; Richardson et al. 1986), ist seine Anwendbarkeit offenbar auf kontinentale Klimate beschränkt (Anderson u. Seeley 1992).

Das in Israel entwickelte Kälteportionen-Modell (auch als Dynamisches Modell bekannt) erscheint bisher am ausgereiftesten (Tabelle 1). Es basiert auf einem komplizierteren Konzept als vorangegangene Ansätze, die lediglich verschiedenen Temperaturbereichen variierende Gewichtungen zuwiesen. Im Kälteportionen-Modell wird angenommen, dass das Chilling in einem zweiphasigen Prozess angesammelt wird. Es entsteht zunächst in einem durch Kälteeinwirkung begünstigten Prozess eine Chilling-Vorstufe, die durch warme Temperaturen wieder abgebaut werden kann (Fishman et al. 1987a,b). Moderate Temperaturen wandeln diese Vorstufe dann in einem irreversiblen Prozess in eine Kälteportion (engl. Chill Portion) um. Im Gegensatz zu seinen Vorläufern erklärt dieses Modell experimentelle Ergebnisse aus Israel (Tabelle 1), mit denen gezeigt wurde, dass Temperaturzyklen unterschiedlicher Frequenz sich verschieden auf die Kälteakkumulation auswirkten, und dass auf niedrige Temperaturen folgende moderate Temperaturen die Ansammlung von Kälteportionen beschleunigen (Erez et al. 1979a, b).
Der Vollständigkeit halber sei hier auch auf ChillingModelle hingewiesen, die vornehmlich in ökologischen und forstwissenschaftlichen Kontexten angewendet werden (z. B. Chuine u. Cour 1999; Linkosalo et al. 2006). Diese Modelle berücksichtigen allerdings zumeist nur die Tagesdurchschnittstemperatur, was angesichts der experimentellen Ergebnisse aus Israel unzureichend erscheint. Dennoch finden in einigen Obstanbaugebieten wie in den US-Staaten Georgia und Florida sogar Modelle Verwendung, die lediglich auf monatlichen Durchschnittstemperaturen basieren (Byrne u. Bacon 2009). Da die meisten dieser Modelle entweder konzeptionelle Schwächen aufweisen oder kaum außerhalb ihrer Herkunftsregion verbreitet sind, beschränken wir uns im Folgenden auf die im Obstbau gängigeren Modelle: das Kältestunden-Modell, das Utah-Modell und das Kälteportionen-Modell.

Ein Vergleich der Auswirkungen unterschiedlicher Temperaturverläufe auf die Kältewirkungs-Ansammlung veranschaulicht die Unterschiede zwischen diesen Modellen. Abbildung 3 zeigt die Anzahl an Kälteeinheiten für die drei wichtigsten Quantifizierungsmethoden, die während einer zweiwöchigen Phase als Folge von sechs unterschiedlichen Temperaturverläufen angesammelt werden. Die Temperaturverläufe sind darin konstante 5 bzw.
Abb. 3 Auswirkung von sechs simulierten Temperaturverläufen (obere Grafik) auf die Chilling-Akkumulation, berechnet nach dem Kältestunden-, Utah- und Kälteportionen-Modell. Die dargestellten Temperaturverläufe sind konstante 5 oder $8^{\circ} \mathrm{C}$ (konstant $5^{\circ} \mathrm{C}$ und konstant $8^{\circ} \mathrm{C}$ ), sowie tägliche Temperaturzyklen mit Tageshöchst- und Tiefstwerten von 8 und $0^{\circ} \mathrm{C}$ (Zyklus $0-8^{\circ} \mathrm{C}$ ), 0 und $22^{\circ} \mathrm{C}$ (Zyklus $\left.0-22^{\circ} \mathrm{C}\right), 5$ und $15^{\circ} \mathrm{C}($ Zyklus $5-15^{\circ} \mathrm{C}$ ) und 5 und $22^{\circ} \mathrm{C}$ (Zyklus $5-22^{\circ} \mathrm{C}$ )
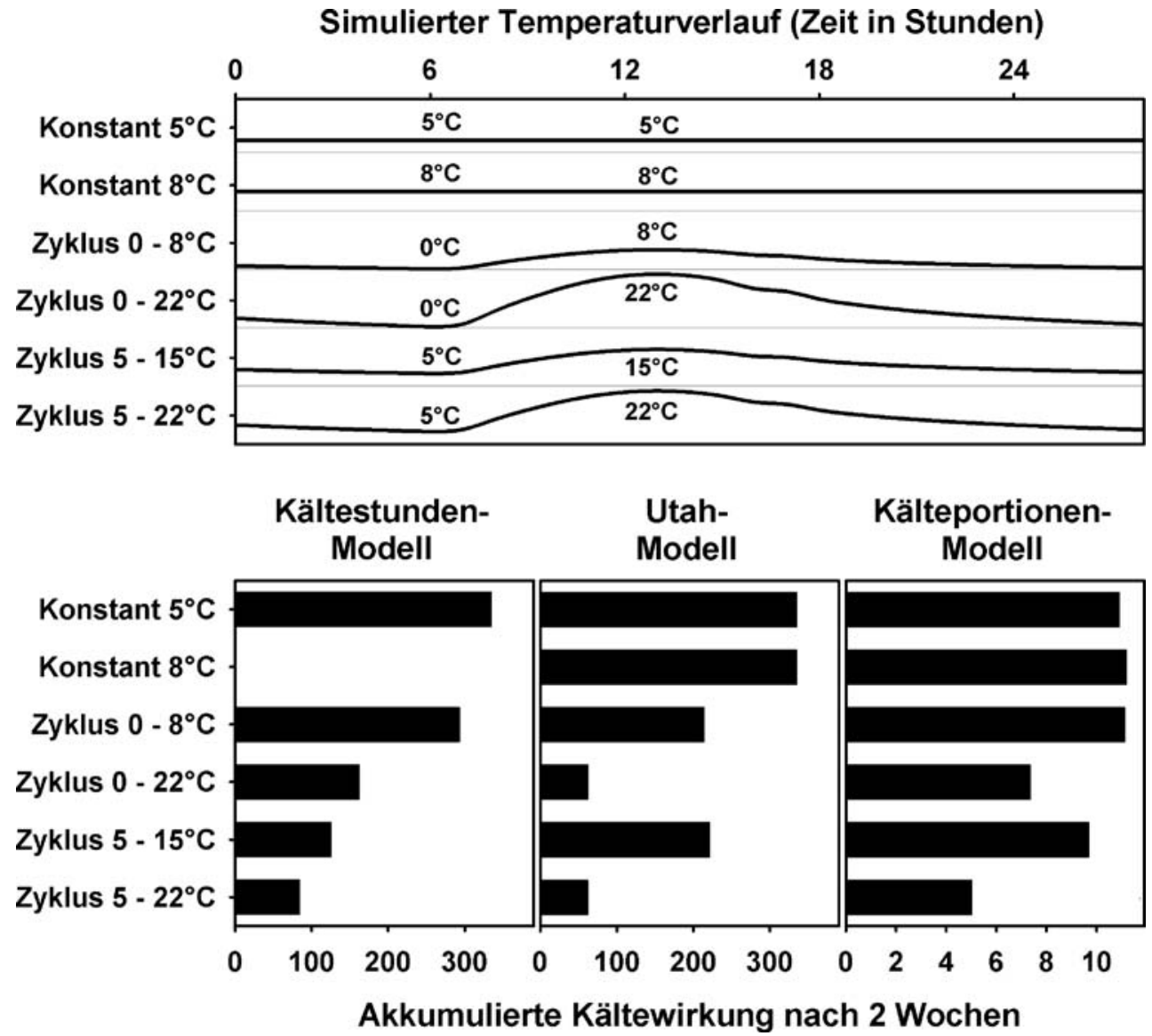
$8^{\circ} \mathrm{C}$ sowie vier simulierte tägliche Temperaturzyklen, in denen die Temperatur zwischen vorgegebenen Tiefst- und Höchstwerten schwankt.

Besonders auffällig ist hierbei, dass im KältestundenModell eine Temperatur von $5^{\circ} \mathrm{C}$ für jede Stunde eine Kältestunde registriert, während schon bei geringfügig höherer Temperatur $\left(8^{\circ} \mathrm{C}\right)$ überhaupt kein Chilling angesammelt wird. Auch die simulierten Temperaturzyklen wirken sich schwächer aus als in den anderen Modellen. Im Utah-Modell werden vor allem die negativen Auswirkungen von Temperaturen über $15,9^{\circ} \mathrm{C}$ auf die Ansammlung von Kältewirkung deutlich. Im Vergleich zu den beiden anderen Modellen zeigt das Kälteportionen-Modell ein sehr viel geringeres Abfallen der Chilling-Effektivität in den Temperaturzyklen, die höhere Temperaturen beinhalten (Abb. 3).

In der Literatur, die sich auf Deutschland bezieht, finden sich bis heute unseres Wissens keine Hinweise auf das Kälteportionen-Modell (Friedrich u. Fischer 2000; Müller u. Braun 2008; Winter et al. 1992). Da dieses Modell jedoch unserer Ansicht nach das theoretisch überzeugendste ist, stellen wir in diesem Artikel Kältewirkung nach dem Kälteportionen-Modell den gängigeren Kältestunden gegenüber. Fischer u. Lüdders (1995) berichten, dass das Kältestunden-Modell zur Einstufung der Anbaueignung für Apfel in den Höhenlagen Kolumbiens ungeeignet ist. Aktuelle Forschungsergebnisse aus Kalifornien haben außerdem gezeigt, dass das Kältestunden-Modell sogar dort, wo es entwickelt wurde, in der Erklärung von WalnussBlühdaten dem Kälteportionen-Modell unterlegen ist (Luedeling et al. 2009c).

\section{Kältewirkung in Deutschland}

Bisher war in Deutschland die Erfüllung des Kältebedürfnisses der heimischen Obstkulturen im Allgemeinen nicht gefährdet. Ob dies jedoch in Zeiten des Klimawandels auf lange Sicht gewährleistet bleibt, scheint angesichts der in den letzten Jahrzehnten stetig ansteigenden Temperaturen fraglich. Auch die Verfrühung der Blühzeitphasen im Meckenheimer Obstanbaugebiet in den letzten 50 Jahren um bis zu 10 Tage verdeutlicht den Einfluss des Klimawandels auf den deutschen Obstbau (Blanke u. Kunz 2009). Intuitiv scheint ein Temperaturanstieg zunächst einmal einen Rückgang an Chilling mit sich zu bringen. Solch ein Rückgang wurde im Laufe der vergangenen Jahre bereits für Kalifornien (Baldocchi u. Wong 2008; Luedeling et al. 2009d,b) und für Hochgebirgsoasen im Oman (Luedeling et al. 2009a) prognostiziert, teilweise mit alarmierenden Rückgangsraten. Dabei stellte sich zunehmend heraus, dass die Geschwindigkeit des Chilling-Rückganges stark von der Wahl des Kältemodells abhängt (Luedeling et al. 2009d,b,c). Das Kälteportionen-Modell ist, zumindest unter subtropischen Bedingungen in Kalifornien, erfolgreicher im Erklären der Obstbaumphänologie als das Kältestunden-Modell (Luedeling et al. 2009c).

Die Zielsetzung dieses Artikels ist es, die Diskussion über die Auswirkungen des Klimawandels auf den Obstanbau in Deutschland mit empirischen Daten zu untermauern. $\mathrm{Zu}$ diesem Zweck haben wir die Auswirkungen historischer Klimaveränderungen in Deutschland auf die Chilling-Akkumulation untersucht. Um die Auswirkungen der Kältemodellwahl zu testen, verwenden wir sowohl das in Deutschland übliche Kältestunden-Modell als auch das theoretisch überzeugendere KälteportionenModell.

\section{Material und Methoden}

\section{Herkunft der Temperaturdaten}

Die Berechnung der historischen Kältewirkung basiert auf Aufzeichnungen der täglichen Höchst- und Tiefsttemperaturen von 43 Wetterstationen in Deutschland (Tabelle 2; Deutscher Wetterdienst 2009). Der am weitesten zurückreichende Datensatz mit Aufzeichnungen seit 1876 ist von der Wetterstation Hohenpeissenberg verfügbar. Für vier Stationen (Münster-Osnabrück, München-Flughafen, Nürburg-Barweiler und Emden-Flugplatz) waren Aufzeichnungen erst seit den 1980er-Jahren vorhanden. Da solch kurze Datenreihen keine verlässliche Aussage über Langzeittrends erlauben, wurden diese Stationen nicht in den präsentierten Klimaänderungskarten berücksichtigt. Viele Stationen wiesen Datenlücken auf, die jedoch im Verhältnis zur Anzahl verfügbarer Tagesdaten relativ klein waren. Für Winter, in denen weniger als 10 Tagesdatensätze fehlten, konnten diese Lücken durch lineare Interpolation geschlossen werden. Winter mit mehr als 10 fehlenden Tagesdatensätzen gingen nicht in die Berechnungen ein.

Da die Berechnung des Chillings mit den üblichen Modellen stündliche Temperaturaufzeichnungen erfordert, war es notwendig, die täglichen Temperaturen mittels idealisierter täglicher Temperaturkurven in stündliche Datensätze umzuwandeln. Nach Linvill (1990) fanden folgende Gleichungen Anwendung:

1. Für den Zeitraum zwischen Sonnenaufgang bis eine Stunde nach Sonnenuntergang $T(t)=\left(T_{\max }-T_{\min }\right)$. $\sin [(\pi \cdot t) /(T L+4)]+T_{\min }$, wobei $T$ die Temperatur zum Zeitpunkt $t$ (in Stunden) nach Sonnenaufgang, $T_{\max }$ und $T_{\min }$ die tägliche Höchst- bzw. Tiefsttemperatur und $T L$ die Tageslänge ist.

2. Für den Zeitraum der Nachtstunden (ab eine Stunde nach Sonnenuntergang) $T(t)=T_{\mathrm{s}}-\left[\left(T_{\mathrm{s}}-T_{\min +1}\right) / \ln (24-\right.$ $T L)] \cdot \ln (t)$, wobei $T_{\mathrm{s}}$ die Temperatur bei Sonnenunter- 
Tabelle 2 Liste der verwendeten Wetterstationen, Beginn der Wetteraufzeichnungen, geografische Lage, durchschnittlich errechnete Kältewirkung $(\varnothing)$ und Veränderungen seit 1950 $\left(\Delta^{\prime} 50 /^{\prime} 10\right)$, quantifiziert nach dem Kältestunden- und dem Kälteportionen-Modell

\begin{tabular}{|c|c|c|c|c|c|c|}
\hline \multirow{2}{*}{$\begin{array}{l}\text { Wetterstation } \\
\text { Aachen }\end{array}$} & \multirow{2}{*}{$\begin{array}{c}\text { Beginn } \\
1891\end{array}$} & \multirow{2}{*}{$\begin{array}{c}\text { Position } \\
50,78^{\circ} \mathrm{N}, 6,1^{\circ} \mathrm{O}, 205 \mathrm{~m}\end{array}$} & \multicolumn{2}{|c|}{$\begin{array}{l}\text { Kältestunden } \\
\varnothing \quad\left(\Delta^{\prime} 50 /^{\prime} 10\right)\end{array}$} & \multicolumn{2}{|c|}{$\begin{array}{l}\text { Kälteportionen } \\
\varnothing \quad\left(\Delta^{\prime} 50 /^{\prime} 10\right)\end{array}$} \\
\hline & & & 2406 & -112 & 144,1 & $-2,5$ \\
\hline Augsburg & 1947 & $48,43^{\circ} \mathrm{N}, 10,93^{\circ} \mathrm{O}, 463 \mathrm{~m}$ & 2290 & 18 & 135,6 & 6,3 \\
\hline Bamberg & 1949 & $49,88^{\circ} \mathrm{N}, 10,92^{\circ} \mathrm{O}, 243 \mathrm{~m}$ & 2402 & -64 & 136,4 & 4,7 \\
\hline Berlin-Tempelhof & 1948 & $52,47^{\circ} \mathrm{N}, 13,4^{\circ} \mathrm{O}, 48 \mathrm{~m}$ & 2426 & -88 & 136,3 & 2,5 \\
\hline Bremen & 1890 & $53,05^{\circ} \mathrm{N}, 8,8^{\circ} \mathrm{O}, 5 \mathrm{~m}$ & 2584 & -133 & 145,5 & $-0,1$ \\
\hline Dresden-Klotzsche & 1967 & $51,13^{\circ} \mathrm{N}, 13,75^{\circ} \mathrm{O}, 232 \mathrm{~m}$ & 2378 & -293 & 136,0 & 1,5 \\
\hline Düsseldorf & 1969 & $51,30^{\circ} \mathrm{N}, 6,77^{\circ} \mathrm{O}, 41 \mathrm{~m}$ & 2329 & -669 & 142,5 & $-11,6$ \\
\hline Emden-Flugplatz & 1997 & $53,38^{\circ} \mathrm{N}, 7,23^{\circ} \mathrm{O}, 5 \mathrm{~m}$ & 2520 & & 149,1 & \\
\hline Erfurt-Bindersleben & 1951 & $50,98^{\circ} \mathrm{N}, 10,97^{\circ} \mathrm{O}, 322 \mathrm{~m}$ & 2361 & 4 & 138,6 & 7,4 \\
\hline Fehmarn & 1948 & $54,53^{\circ} \mathrm{N}, 11,07^{\circ} \mathrm{O}, 9 \mathrm{~m}$ & 2900 & -23 & 141,9 & 5,0 \\
\hline Fichtelberg & 1947 & $50,43^{\circ} \mathrm{N}, 12,95^{\circ} \mathrm{O}, 1215 \mathrm{~m}$ & 1540 & 269 & 138,0 & 6,3 \\
\hline Frankfurt/M-Flughafen & 1949 & $50,05^{\circ} \mathrm{N}, 8,6^{\circ} \mathrm{O}, 113 \mathrm{~m}$ & 2364 & -214 & 137,8 & $-0,2$ \\
\hline Görlitz & 1947 & $51,17^{\circ} \mathrm{N}, 14,95^{\circ} \mathrm{O}, 240 \mathrm{~m}$ & 2371 & -23 & 134,8 & 3,5 \\
\hline Greifswald & 1947 & $54,10^{\circ} \mathrm{N}, 13,4^{\circ} \mathrm{O}, 2 \mathrm{~m}$ & 2610 & -47 & 140,9 & 3,1 \\
\hline Hamburg-Fuhlsbüttel & 1891 & $53,63^{\circ} \mathrm{N}, 9,98^{\circ} \mathrm{O}, 15 \mathrm{~m}$ & 2617 & -167 & 144,3 & $-0,1$ \\
\hline Hannover & 1936 & $52,47^{\circ} \mathrm{N}, 9,68^{\circ} \mathrm{O}, 59 \mathrm{~m}$ & 2441 & -84 & 142,4 & 2,6 \\
\hline Helgoland & 1952 & $54,18^{\circ} \mathrm{N}, 7,9^{\circ} \mathrm{O}, 4 \mathrm{~m}$ & 2995 & -229 & 149,8 & 5,9 \\
\hline Hof & 1947 & $50,32^{\circ} \mathrm{N}, 11,88^{\circ} \mathrm{O}, 568 \mathrm{~m}$ & 2233 & 222 & 136,7 & 5,9 \\
\hline Hohenpeissenberg & 1876 & $47,80^{\circ} \mathrm{N}, 11,02^{\circ} \mathrm{O}, 986 \mathrm{~m}$ & 2082 & -14 & 147,6 & $-2,6$ \\
\hline Kahler Asten & 1950 & $51,18^{\circ} \mathrm{N}, 8,48^{\circ} \mathrm{O}, 859 \mathrm{~m}$ & 2139 & 447 & 148,9 & 7,7 \\
\hline Karlsruhe & 1876 & $49,03^{\circ} \mathrm{N}, 8,37^{\circ} \mathrm{O}, 145 \mathrm{~m}$ & 2300 & -91 & 136,2 & $-4,7$ \\
\hline Kempten & 1952 & $47,72^{\circ} \mathrm{N}, 10,33^{\circ} \mathrm{O}, 705 \mathrm{~m}$ & 2177 & 91 & 142,1 & $-1,4$ \\
\hline Konstanz & 1970 & $47,68^{\circ} \mathrm{N}, 9,18^{\circ} \mathrm{O}, 447 \mathrm{~m}$ & 2432 & -797 & 135,9 & $-3,5$ \\
\hline Leipzig-Schkeuditz & 1972 & $51,42^{\circ} \mathrm{N}, 12,23^{\circ} \mathrm{O}, 149 \mathrm{~m}$ & 2429 & -666 & 139,0 & $-12,8$ \\
\hline Lindenberg & 1947 & $52,22^{\circ} \mathrm{N}, 14,12^{\circ} \mathrm{O}, 98 \mathrm{~m}$ & 2376 & -4 & 134,7 & 4,3 \\
\hline List auf Sylt & 1937 & $55,02^{\circ} \mathrm{N}, 8,42^{\circ} \mathrm{O}, 29 \mathrm{~m}$ & 2914 & 75 & 147,4 & 6,9 \\
\hline Magdeburg & 1947 & $52,13^{\circ} \mathrm{N}, 11,6^{\circ} \mathrm{O}, 85 \mathrm{~m}$ & 2437 & -51 & 137,5 & 4,9 \\
\hline Meiningen & 1979 & $50,57^{\circ} \mathrm{N}, 10,38^{\circ} \mathrm{O}, 453 \mathrm{~m}$ & 2360 & 372 & 140,8 & 12,2 \\
\hline München-Flughafen & 1992 & $48,37^{\circ} \mathrm{N}, 11,8^{\circ} \mathrm{O}, 447 \mathrm{~m}$ & 2172 & & 134,7 & \\
\hline Münster-Osnabrück & 1980 & $52,13^{\circ} \mathrm{N}, 7,7^{\circ} \mathrm{O}, 53 \mathrm{~m}$ & 2358 & & 144,2 & \\
\hline Neuruppin & 1961 & $52,90^{\circ} \mathrm{N}, 12,82^{\circ} \mathrm{O}, 39 \mathrm{~m}$ & 2498 & 141 & 137,5 & 10,6 \\
\hline Nürburg-Barweiler & 1995 & $50,35^{\circ} \mathrm{N}, 6,87^{\circ} \mathrm{O}, 486 \mathrm{~m}$ & 2476 & & 149,0 & \\
\hline Nürnberg & 1955 & $49,50^{\circ} \mathrm{N}, 11,05^{\circ} \mathrm{O}, 318 \mathrm{~m}$ & 2351 & -9 & 136,4 & 2,6 \\
\hline Potsdam & 1893 & $52,38^{\circ} \mathrm{N}, 13,07^{\circ} \mathrm{O}, 81 \mathrm{~m}$ & 2399 & -39 & 135,4 & $-0,9$ \\
\hline Rostock-Warnemünde & 1947 & $54,18^{\circ} \mathrm{N}, 12,08^{\circ} \mathrm{O}, 10 \mathrm{~m}$ & 2777 & -84 & 142,4 & 5,2 \\
\hline Saarbrücken-Ensheim & 1951 & $49,22^{\circ} \mathrm{N}, 7,12^{\circ} \mathrm{O}, 320 \mathrm{~m}$ & 2424 & -81 & 142,6 & 1,1 \\
\hline Schleswig & 1947 & $54,53^{\circ} \mathrm{N}, 9,55^{\circ} \mathrm{O}, 48 \mathrm{~m}$ & 2738 & 35 & 146,8 & 6,2 \\
\hline Schwerin & 1890 & $53,65^{\circ} \mathrm{N}, 11,38^{\circ} \mathrm{O}, 68 \mathrm{~m}$ & 2623 & -75 & 140,8 & 0,9 \\
\hline Straubing & 1951 & $48,83^{\circ} \mathrm{N}, 12,57^{\circ} \mathrm{O}, 359 \mathrm{~m}$ & 2240 & 24 & 128,3 & 11,3 \\
\hline Stuttgart-Echterdingen & 1953 & $48,68^{\circ} \mathrm{N}, 9,23^{\circ} \mathrm{O}, 391 \mathrm{~m}$ & 2333 & -106 & 138,7 & 0,5 \\
\hline Trier-Petrisberg & 1948 & $49,75^{\circ} \mathrm{N}, 6,67^{\circ} \mathrm{O}, 273 \mathrm{~m}$ & 2434 & -140 & 141,3 & 0,4 \\
\hline Würzburg & 1947 & $49,77^{\circ} \mathrm{N}, 9,97^{\circ} \mathrm{O}, 272 \mathrm{~m}$ & 2374 & -29 & 135,9 & 4,6 \\
\hline Zugspitze & 1900 & $47,42^{\circ} \mathrm{N}, 10,98^{\circ} \mathrm{O}, 2962 \mathrm{~m}$ & 316 & 138 & 78,2 & 18,5 \\
\hline
\end{tabular}

gang, $t$ die Zeit nach Sonnenuntergang und $T_{\text {min+1 }}$ die Tiefsttemperatur des folgenden Tages ist.

Aus den mit diesen Formeln ermittelten stündlichen Temperaturwerten berechneten wir dann die ChillingAkkumulation nach dem Kältestunden- und KälteportionenModell. Um Kältewirkung nach dem Kältestunden-Modell zu berechnen, müssen ein Anfangs- und ein End-Datum für die Wintersaison festgelegt werden. Hierfür wurden der 1. Oktober und der 1. Mai verwendet. Das Kälteportionen-
Modell enthält einen Selbstregulierungsmechanismus, der die Ansammlung von Chilling während der warmen Monate verhindert. Daher wurde das Anfangsdatum für die Kälteportions-Ansammlung auf den 1. Juli gesetzt.

Als Kältestunden wurden alle Stunden gewertet, in denen Temperaturen zwischen 0 und $7,2^{\circ} \mathrm{C}$ vorherrschten. Die Gleichungen für die Berechnungen der Kälteportionen sind zu komplex, um sie hier darzustellen. Sie können aber bei Luedeling et al. (2009b) nachgelesen werden. Die Ermittlung der beiden Kältemetriken für alle Winter, in denen 
Temperaturaufzeichnungen vorlagen bzw. ermittelt werden konnten, erfolgte mit der Programmiersprache JSL (JMP Scripting Language; JMP 7, SAS Inst., Cary, NC, USA).

\section{Zeitliche Trends und räumliche Interpolation}

Zur Ermittlung langzeitlicher Trends wurden mit beiden Modellen für alle verfügbaren Stationen mittels linearer Regression die jährlichen Raten bestimmt, mit denen sich die Kältewirkung im Laufe der Jahre verändert hat. Diese Ergebnisse erlaubten die Extrapolation des historischen Datensatzes, um das voraussichtliche Chilling für alle Stationen im Jahr 2010 abzuschätzen. Ebenfalls ließen diese Ergebnisse die Abschätzung rezenter Chilling-Verluste zwischen 1950 und $2010 \mathrm{zu}$.

Durch räumliche Interpolation (ArcGIS 9.2, ESRI, Redlands, CA, USA) entstand aus den Daten der einzelnen Wetterstationen eine flächendeckende Chilling-Karte für Deutschland. Diese Interpolation erfolgte mit der KrigingInterpolationstechnik. Da viele Regionen Deutschlands jedoch weit von der nächsten Wetterstation entfernt liegen und die unterschiedlichen Höhenlagen der Mittelgebirge und die geographische Breite sich ebenfalls entscheidend auf die Temperaturverläufe auswirken, war es nötig, einen Korrekturmechanismus für diese Variation in die Interpolation einzubauen. Sowohl für die Höhenlage als auch für die geographische Breite berechneten wir hierzu zunächst die Differenzen zwischen den wahren Werten für jeden Punkt in Deutschland (Höhenlage nach dem SRTM30Höhenmodell der Global Land Cover Facility, University of Maryland, USA) und durch die Positionen der Wetterstationen interpolierter Oberflächen für Breite und Höhe. Diese Differenzoberflächen repräsentierten den zusätzlichen Einfluss der Höhe und Breite, der nicht durch einfache Interpolation der berechneten Chilling-Werte abgedeckt wurde. Um diesen Anteil ebenfalls zu berücksichtigen, berechneten wir mittels PLS-Regression den Einfluss des Zusammenwirkens von Höhenlage und Breitengrad auf die Kältewirkung, multiplizierten die Differenzoberflächen mit den jeweiligen Zuwachsraten und addierten diese Oberfläche auf die interpolierte Chilling-Oberfläche.

Dieses Verfahren wurde sowohl für die repräsentativ geschätzte Kältewirkung im Jahr 2010 als auch für die Chilling-Veränderungen zwischen 1950 und 2010 angewendet.

\section{Ergebnisse}

Deutschlandweite verfügbare Kältewirkung und zeitliche Trends

Die nach dem Kältestunden-Modell berechnete Kältewirkung lässt über den Zeitraum, für den Wetteraufzeichnungen vorlagen, deutschlandweit keinen signifikanten Trend erkennen (Abb. 4). Die Regression über alle Abschätzungen der Kältestunden der einzelnen Wetterstationen ergab nur einen Rückgang um 0,06 Kältestunden pro Jahr bzw. 0,002\% des langjährigen Mittelwerts (2423 Kältestunden). Die Jahre mit den wenigsten Kältestunden waren hierbei 1963 (1699 Kältestunden), 1996 (1721), 1947 (1831) und 1970 (1843). Die meisten Kältestunden standen 1975 (3249 Kältestunden), 1898 (3110), 1923 (3084) und 1905 (2874) zur Verfügung. Bei dieser Betrachtung muss allerdings beachtet werden, dass die Anzahl der Wetterstationen, die in die Berechnung des landesweiten Durchschnitts eingingen, teilweise stark unterschiedlich war (Abb. 4, Balkendiagramm).

Wird das Kälteportionen-Modell für die Berechnung verwendet, ergibt sich ein leicht stärkerer, aber dennoch nicht signifikant abnehmender Chilling-Trend um 0,04 Kälteportionen pro Jahr bzw. 0,03\% des langjährigen Durchschnitts von 141,5 Kälteportionen (Abb. 5). Die Jahre mit der geringsten Kältewirkung waren 1996 (113,7 Kälteportionen), 1947 (116,5), 1963 (118,2) und 1970 (120,1). Die meisten Kälteportionen standen 1913 (167,0 Kälteportionen), 1975 $(162,7), 1884$ und 1883 (jeweils 159,5) zur Verfügung.

Regionale Unterschiede und zeitliche Trends der Kältewirkung in Deutschland

Innerhalb Deutschlands konnten große Unterschiede in der Chilling-Verfügbarkeit festgestellt werden. Nach dem Kältestunden-Modell ergab sich in der Prognose für 2010 vor allem ein Nord-Süd-Gefälle, wobei die meisten Kältestunden im Norden und die wenigsten im Süden auftraten (Schleswig-Holstein mit 3000 Kältestunden, Alpenrand nur etwa 1750 Kältestunden). Viele Kältestunden ergaben sich auch im Rhein-Mosel-Gebiet mit über 2500 Kältestunden (Abb. 6a). Darüber hinaus ließ sich ein starker Einfluss der Höhenlage feststellen. Auf den Gipfeln des Fichtelberges und der Zugspitze wurden zum Beispiel sehr wenige Kältestunden ermittelt (Tabelle 2).

Nach dem Kälteportionen-Modell ergab sich ein leicht anderes Muster (Abb. 6b). Das Gebiet mit den meisten Kälteportionen erstreckt sich von Schleswig-Holstein und Ostfriesland in einem langen Streifen bis an Rhein und Mosel. Hier sind zwischen 145 und 150 Kälteportionen typisch. Verhältnismäßig wenige Kälteportionen sind nach diesem Modell in Südwestdeutschland und Südbayern zu erwarten. Auch in Sachsen, Thüringen, weiten Teilen Brandenburgs sowie den übrigen Gebieten Bayerns und Baden-Württembergs sind nur etwa 135-140 Kälteportionen typisch. Insgesamt bewegen sich die verfügbaren Kälteportionen (mit Ausnahme der Berggipfel) in einem relativ engen Bereich zwischen 120 und 150 (Tabelle 2). 
Abb. 4 Deutschlandweite jährliche Mittelwerte der Kältestunden über alle verwendeten Wetterstationen und lineare Regression über die Zeit. Die grauen Box-Plots stellen die gesamte Verteilung über alle Stationen dar. Das Balkendiagramm am unteren Rand zeigt die Anzahl der verwendeten Stationen pro Jahr

Abb. 5 Deutschlandweite jährliche Mittelwerte der Kälteportionen über alle verwendeten Wetterstationen und lineare Regression über die Zeit. Die grauen Box-Plots stellen die gesamte Verteilung über alle Stationen dar. Das Balkendiagramm am unteren Rand zeigt die Anzahl der verwendeten Stationen pro Jahr
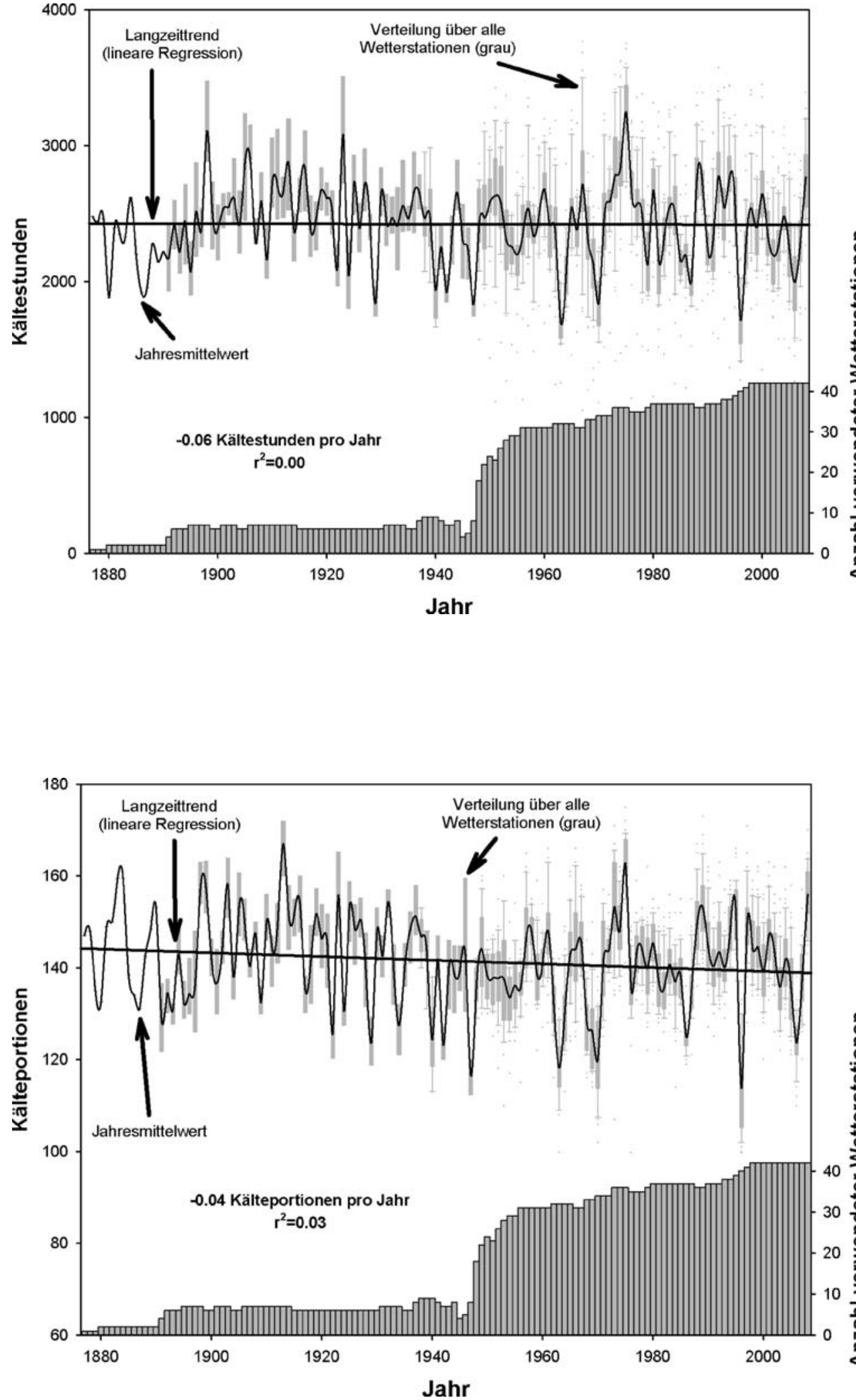
Abb. 6 Unter den Klimabedingungen von $2010 \mathrm{zu}$ erwartende Kältewirkung in Deutschland, quantifiziert in Kältestunden (a) und Kälteportionen (b)

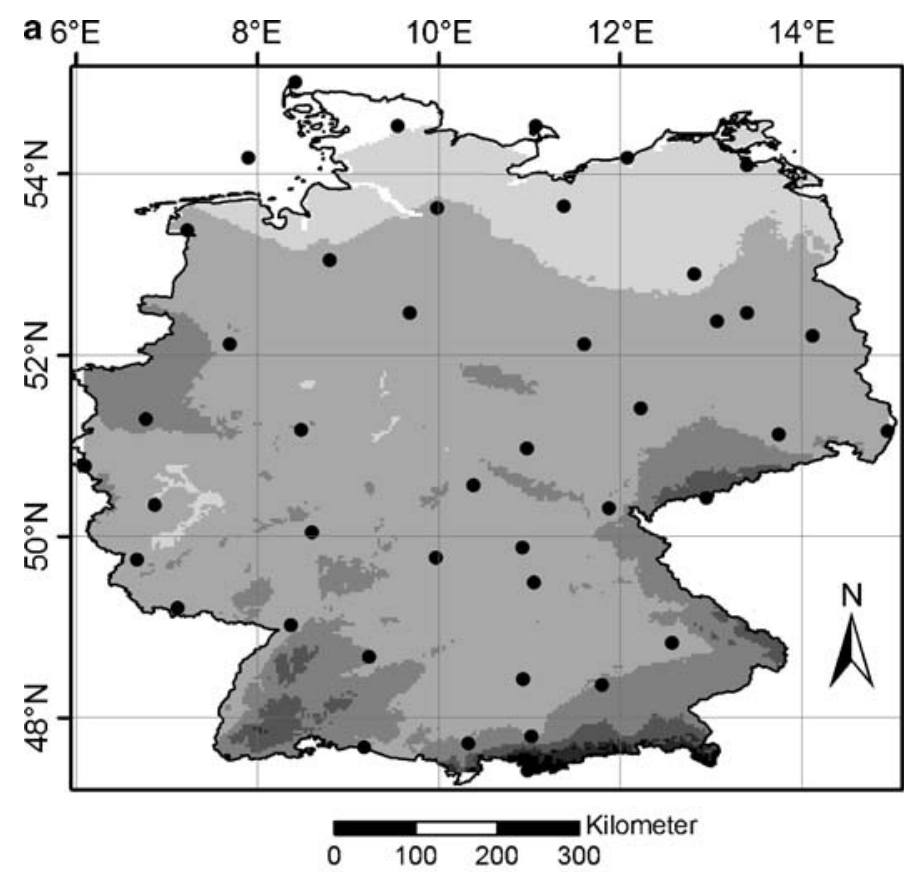

Kältestunden 2010

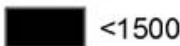

$1500-1750$

$1750-2000$

$2000-2250$

$2250-2500$

$2500-2750$

$2750-3000$

Wetterstation

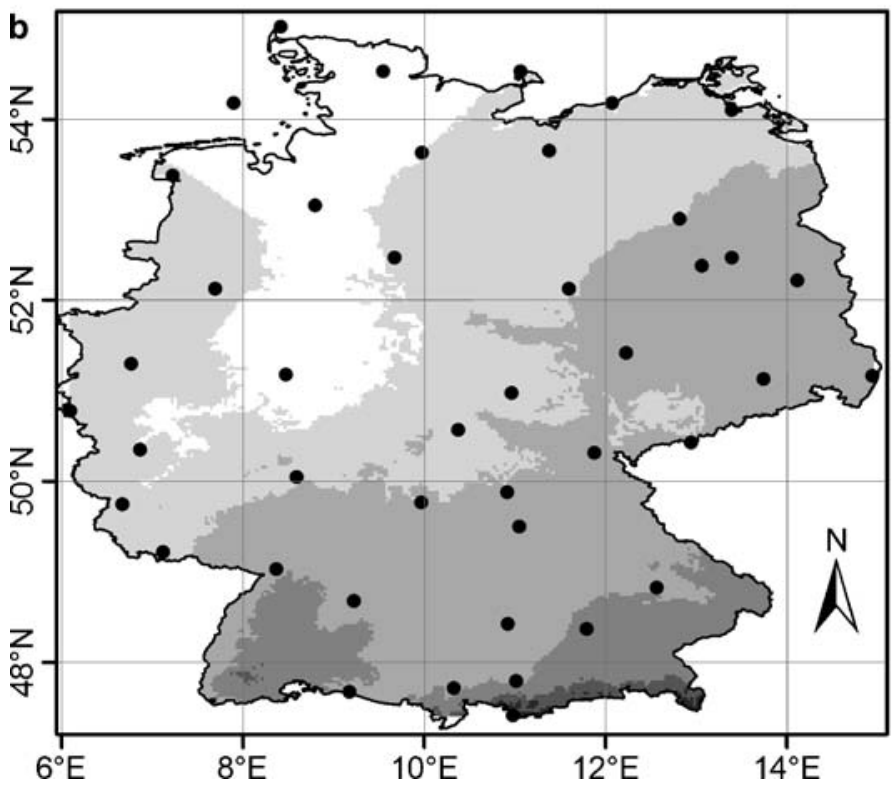

Kälteportionen 2010

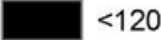

$120-125$

$125-130$

$130-135$

$\square 135-140$

$\square 140-145$

$145-150$

Wetterstation
Der Vergleich zwischen der zu erwartenden Kältewirkung im Jahr 2010 und den typischen Bedingungen um 1950 zeigt, dass sich auch im langzeitlichen Trend starke regionale Unterschiede erkennen lassen (Abb. 7a). Entscheidet man sich für das Kältestunden-Modell, stechen vier Wetterstationen hervor, an denen die Zahl der Kältestunden in diesem Zeitraum stark abgenommen hat (Konstanz -796, Düsseldorf -669, Leipzig-Schkeuditz-666 und Dresden-Klotzsche -293; vgl. Tabelle 2). Anderseits nahmen die Kältestunden auf dem Kahlen Asten (+447) und in Meiningen $(+372)$ stark zu. Außerhalb relativ kleiner Gebiete in der Umgebung der erwähnten Wettersta- tionen änderte sich die Kältewirkung um weniger als 200 Kältestunden gegenüber 1950 (Abb. 7b).

Die gleichen Berechnungen nach dem KälteportionenModell ergaben weit weniger dramatische Veränderungen (Abb. 7b). Die größten Kälteportionenverluste wurden für Leipzig-Schkeuditz $(-12,8)$ und Düsseldorf $(-11,6)$ festgestellt. Auf dem dritten Rang folgte Karlsruhe mit einem sehr viel geringeren Verlust von 4,7 Kälteportionen. Gleichzeitig nahm an einigen Standorten die Kältewirkung stark zu (Meiningen +12,2, Straubing +11,3 und Neuruppin $+10,6)$. Im größten Teil Deutschlands schwankten die Chilling-Veränderungen in diesem Zeitraum zwischen 
Abb. 7 Veränderungen in der Kältewirkungsverfügbarkeit zwischen 1950 und 2010, basierend auf langzeitlichen Trends, quantifiziert in Kältestunden (a) und Kälteportionen (b)
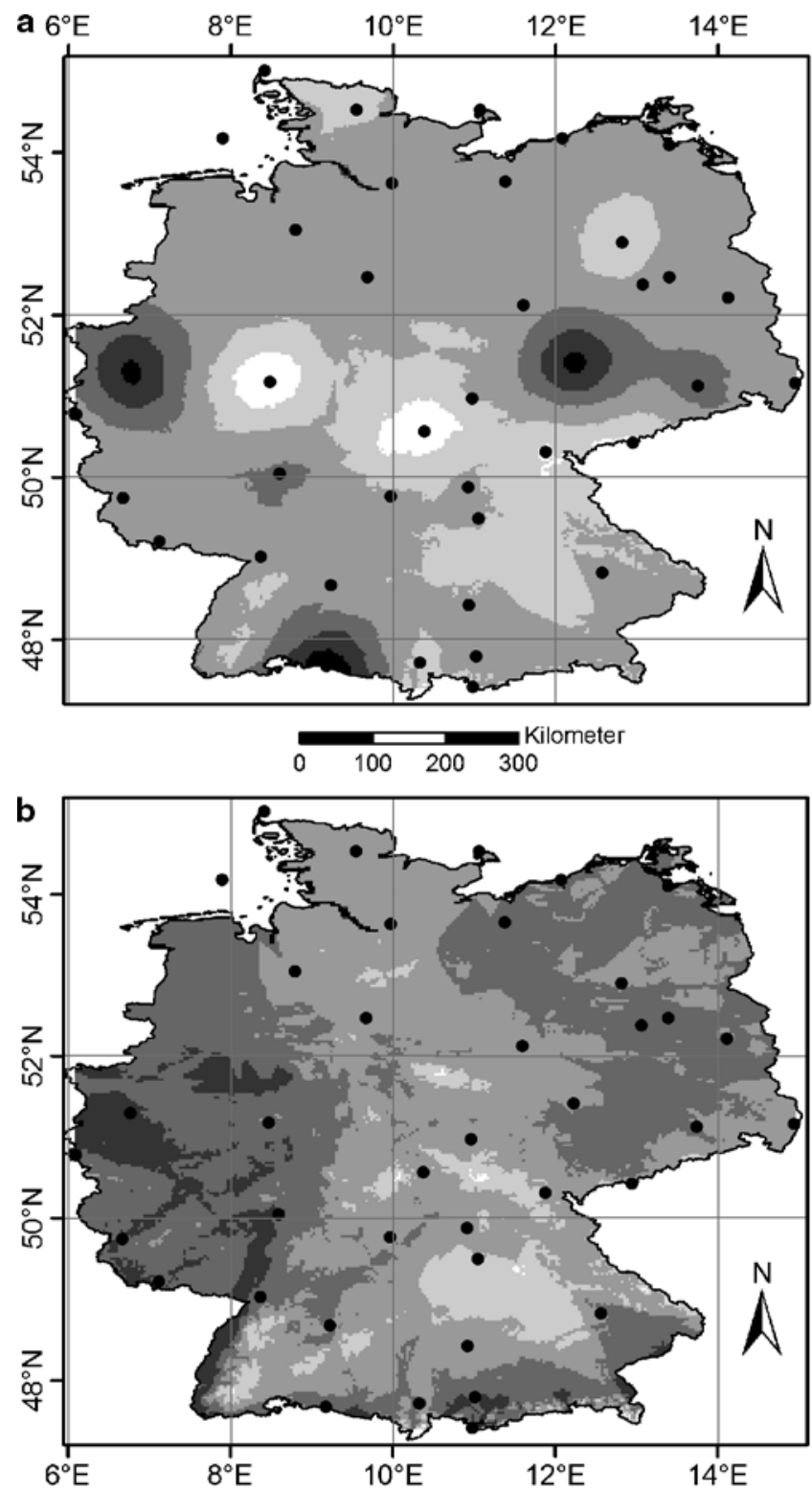

Veränderung 1950 - 2010 Kältestunden

$<<-600$

$-600--400$

$-400--200$

$-200-0$

0 - +200

$>+200$

\section{Wetterstation}

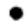

Veränderung 1950 - 2010 Kälteportionen

$<-2.5$

$-2.5-0$

$0-+2.5$

$+2.5-+5$

$+5-+7.5$

$>+7.5$

\section{Wetterstation}

$+2,5$ und $-2,5$ Kälteportionen. Prozentual ausgedrückt variierten die Chilling-Veränderungen im untersuchten 60-Jahre-Zeitraum von -27 bis $+24 \%$ für das KältestundenModell und von -9 bis $+9 \%$ für das Kälteportionen-Modell.

\section{Diskussion}

Kältewirkung in Deutschland

In Deutschland liegen die Regionen mit dem meisten Chilling an der Nordseeküste und in der Norddeutschen Tiefebene, wo relativ milde Wintertemperaturen vorherrschen und nicht in den südlicheren Gebieten mit ihren oft sehr viel kälteren Wintern. Dies liegt daran, dass in beiden verwende- ten Kältemodellen sowie auch im Utah-Modell, das hier ausgespart blieb, Temperaturen unter dem Gefrierpunkt nicht zur Ansammlung von Kältewirkung beitragen. Somit ergibt sich nach beiden Modellen die geringste Kältewirkung auf der Zugspitze. Generell scheint das verfügbare Chilling negativ mit der Frosthäufigkeit zu korrelieren, weil bei den für Deutschland typischen Wintertemperaturen der Großteil der frostfreien Stunden in den effektivsten Temperaturbereich beider Modelle fällt.

Unter den bedeutenden Obstanbauregionen Deutschlands ergab sich für das Alte Land und die Meckenheimer Gegend verhältnismäßig viel Chilling (etwa 2450 Kältestunden oder 145 Kälteportionen), während die Gegend um Dresden bzw. Halle (2250/138) und die Bodenseeregion (2250/136) etwas weniger Kältewirkung aufweisen. 


\section{Langjährige Trends in der Kältewirkung}

Deutschlandweit lässt sich bisher kaum ein Trend in der Chilling-Verfügbarkeit feststellen. In subtropischen Gebieten, wie z. B. im Hochgebirge des Oman (Luedeling et al. 2009a) oder in Kalifornien (Baldocchi u. Wong 2008; Luedeling et al. 2009a; Luedeling et al. 2009b), wo in den letzten Jahren bereits massive Rückgänge im Chilling verzeichnet wurden, wird es hingegen möglicherweise in der nahen Zukunft zu großen Problemen kommen. Die Ursache für diese regionalen Unterschiede besteht darin, dass die durchschnittlichen Wintertemperaturen in Deutschland deutlich niedriger sind als z. B. in Kalifornien. Dort führt ein leichter Temperaturanstieg dazu, dass mehr Stunden aus dem optimalen Temperaturbereich herausfallen als hinzukommen. In Deutschland ist die Situation umgekehrt. Hier dürften in vielen Fällen mehr Stunden aus dem leichten Frostbereich in den positiven Temperaturbereich rücken als über den oberen Schwellenwert für die ChillingAkkumulation hinauswandern. Das sollte insbesondere beim Kälteportionen-Modell der Fall sein, da es hier keine harte Abbruchkante bei $7,2^{\circ} \mathrm{C}$ gibt und Temperaturen bis etwa $15^{\circ} \mathrm{C}$ kältewirksam sind. Dieser Schwellenwert wird in deutschen Wintern nur sehr selten überschritten. Der Unterschied in den Temperaturobergrenzen beider Modelle erklärt auch, warum die Veränderungen in Kälteportionen $(-9$ bis $+9 \%)$ prozentual sehr viel geringer ausfallen als in Kältestunden $(-27$ bis $+24 \%)$.

Regional ergaben sich große Unterschiede in der Chilling-Entwicklung (Tabelle 3). Nach dem KältestundenModell scheint in den Gegenden um Dresden, Düsseldorf, Konstanz und Leipzig in den letzten 60 Jahren Einiges an Kältestunden verloren gegangen zu sein. Ansonsten gab es nur geringe Verluste bzw. Zunahmen. In Kälteportionen ausgedrückt erscheint die Lage nirgendwo in Deutschland besonders dramatisch.

Sollte sich das Kältestunden-Modell für Deutschland als geeignet erweisen, sieht es so aus, als müssten sich die Obstbauern in der Dresdener Gegend und vor allem am Bodensee in der Zukunft auf deutlich geringere Kältewirkung einstellen. Das Alte Land und die Meckenheimer Gegend scheinen dagegen relativ verschont zu bleiben. Nach dem Kälteportionen-Modell gibt es in keinem der bedeutenden Anbaugebiete Anlass zur Sorge.
Vergleich der Kältemodelle

Die oben angeführten Ergebnisse haben gezeigt, dass es große Unterschiede zwischen den beiden Kältemodellen gibt. Das Kältestunden-Modell wies sowohl in der räumlichen Verteilung als auch in der zeitlichen Entwicklung eine sehr viel stärkere Streuung der Kältewirkung auf als das Kälteportionen-Modell. Das deutet schon an, dass die beiden Modelle offenbar nicht dasselbe messen, und dass eines der Modelle vermutlich die Kältewirkung genauer quantifiziert als das andere. In diesem Zusammenhang lohnt sich ein Blick auf das Verhältnis von Kältestunden zu Kälteportionen. Wäre es sowohl über die Zeit als auch von Ort zu Ort einigermaßen konstant, könnte angenommen werden, dass die Modelle äquivalent sind und etwa gleich nützlich. Abbildung 8 zeigt aber, dass zeitliche Konstanz des Quotienten zwischen den beiden Kältemetriken über die Dauer der hier verwendeten Wetteraufzeichnungen nicht gegeben ist.

Auch der Ort, an dem die Messungen durchgeführt wurden, scheint eine nicht unerhebliche Rolle zu spielen, wobei insbesondere der Breitengrad und die Höhenlage offenbar das Verhältnis von Kältestunden zu Kälteportionen beeinflussen (Abb. 9).

Eine Entscheidung für eines der beiden Modelle für Deutschland lässt sich ohne weitere Untersuchungen nicht treffen. In Kalifornien hat sich vor Kurzem herausgestellt, dass das Kälteportionen-Modell eine sehr viel überzeugendere Erklärung für die historischen Blühzeitpunkte mehrerer Walnusssorten lieferte als der Kältestunden-Ansatz (Luedeling et al. 2009c). Vergleichbare Studien für Deutschland sind uns nicht bekannt.

\section{Auswirkungen verringerter Kältewirkung}

In einigen Anbaugebieten Deutschlands scheinen durchaus von Zeit zu Zeit Jahre mit verhältnismäßig geringer Kältewirkung vorzukommen. In Abhängigkeit vom Kältemodell könnte es in den Anbaugebieten um Dresden und am Bodensee einen langjährigen Trend abnehmenden Chillings geben. Die entscheidende Frage ist jedoch, ob das in der Zukunft negative Folgen für den Obstanbau in Deutschland haben wird. Zweifelsfrei lässt sich momentan noch keine Antwort geben, weil zu wenig über die Kältebedürfnisse
Tabelle 3 Verfügbarkeit und Veränderungen seit 1950 in der Kältewirkung in verschiedenen Obstanbauregionen in Deutschland

\begin{tabular}{lcccc}
\hline Obstanbauregion & \multicolumn{2}{c}{ Kältewirkung um 2010 } & \multicolumn{2}{c}{ Veränderung seit 1950 } \\
& Kältestunden & Kälteportionen & Kältestunden & Kälteportionen \\
\hline Jork, Altes Land & $2400-2600$ & $145-150$ & $-90--100$ & $+3-+4$ \\
Meckenheim, Köln/Bonn & $2300-2500$ & $140-145$ & $-300--500$ & $-0--1$ \\
Dresden, Sachsen & $2100-2300$ & $135-140$ & $-250--300$ & $+2-+3$ \\
Bodensee & $2100-2300$ & $135-140$ & $-600--800$ & $-1-+2$ \\
\hline
\end{tabular}


Abb. 8 Veränderung des deutschlandweit berechneten Quotienten zwischen Kältestunden und Kälteportionen seit 1877
Abb.9 Auswirkung von Breitengrad (linke Grafik) und Meereshöhe (rechte Grafik) auf das Verhältnis von Kältestunden zu Kälteportionen
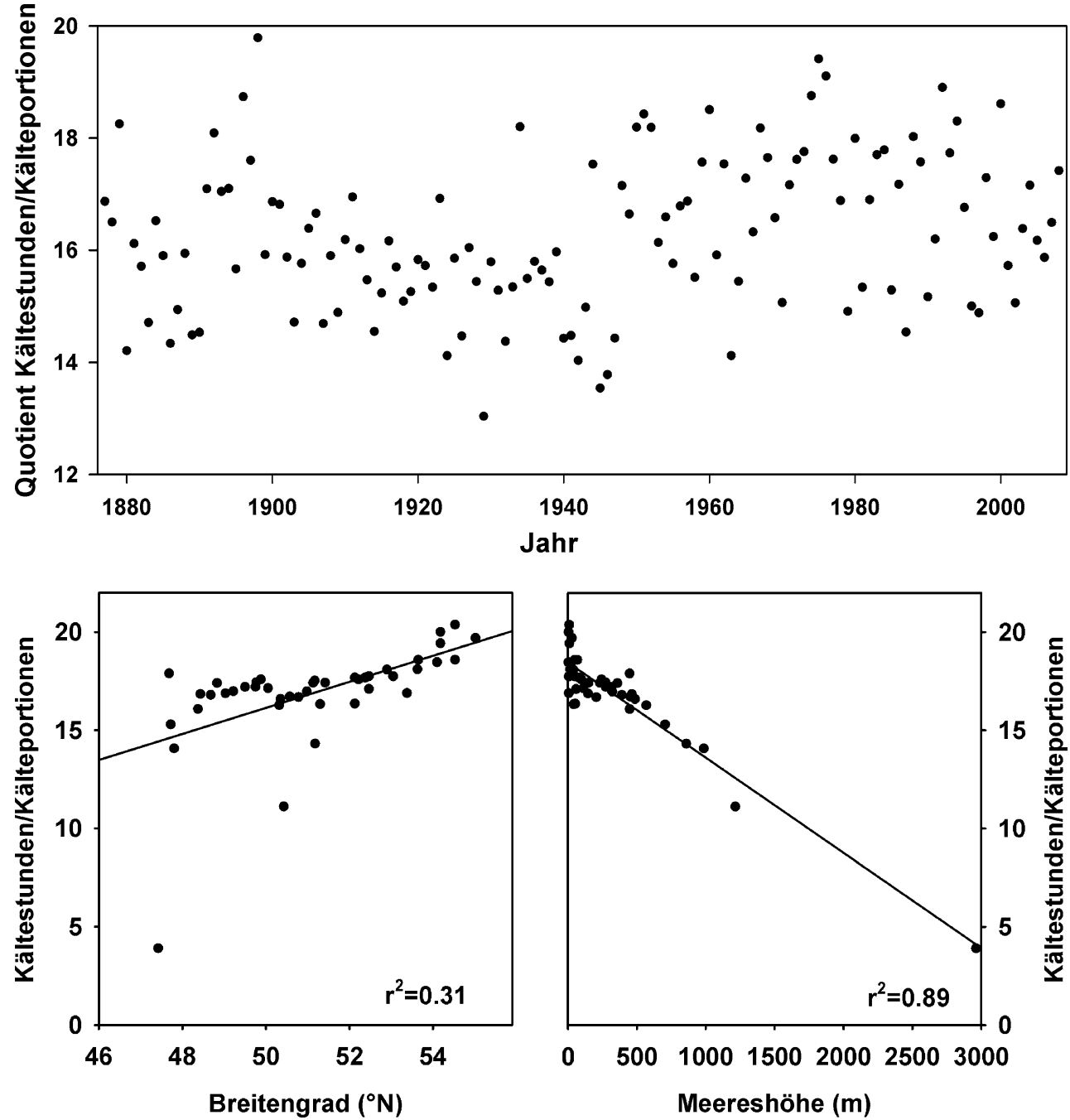

der hiesigen Obstarten und -sorten bekannt ist. Selbst wenn derartige Informationen vorliegen, sind die Angaben nur in Kältestunden zu bekommen. Darüber hinaus wurden sie bisher meist unter kontrollierten, konstanten Temperaturbedingungen ermittelt - eine Methode, die zu zweifelhaften Ergebnissen führen kann (Luedeling et al. 2009c). In Kalifornien sind für viele Obstarten aufgrund des marginalen Winterklimas sehr viel mehr Informationen verfügbar. Hier lässt sich einigermaßen sicher sagen, dass viele Sorten in Zukunft Schwierigkeiten haben werden, ihre Kältebedürfnisse zu befriedigen (Luedeling et al. 2009d). Für Deutschland gibt es jedoch Grund zu der Annahme, dass der Klimawandel zunächst keine direkten negativen Auswirkungen auf das Chilling und damit auf die Erfüllung der Kältebedürfnisse der meisten Obstarten haben wird. Dieser Eindruck ergibt sich aus den Beobachtungen der letzten Jahre. Im warmen Winter 2006/07 mit relativ hohen Temperaturen im Januar wurde eine stark verfrühte, schwache und unregelmäßige Blüte bei vielen Obstgehölzen in Deutschland beobachtet (eigene Beobachtungen, unveröffentlicht). Diese Symptome werden oft mit einer Nichterfüllung des Kältebedürfnisses in Verbindung gebracht. Die Betrachtung der Kältewirkung in diesem Winter bestätigt den Verdacht allerdings nicht, da mit einem deutschlandweiten Mittelwert von 2293 Kältestunden bzw. 137 Kälteportionen eine vollkommen normale Kältewirkung verfügbar war. Der Grund für den veränderten Blühablauf scheint eher darin zu liegen, dass viele Obstgehölze bereits im Januar ihr Kältebedürfnis erfüllt hatten und auf warme Temperaturen reagieren konnten.

Die Brechung der Dormanz erfolgt nach allgemeinem Verständnis in einem zweiphasigen Prozess, in dem zunächst das Kältebedürfnis und anschließend das Wärmebedürfnis zum Erreichen eines bestimmten phänologischen Stadiums erfüllt werden muss (Luedeling et al. 2009c). Nach diesem (möglicherweise leicht vereinfachten) Konzept sollten sowohl Wärme, die vor dem Erfüllen des Kältebedüfnisses auftritt, als auch Kälte (abgesehen von 


\begin{abstract}
Abb. 10 Deutschlandweite jährliche Mittelwerte der Wachstums-Grad-Stunden über alle verwendeten Wetterstationen und lineare Regression über die Zeit. Die grauen Box-Plots stellen die gesamte Verteilung über alle Stationen dar. Das Balkendiagramm am unteren Rand zeigt die Anzahl der verwendeten Stationen pro Jahr
\end{abstract}

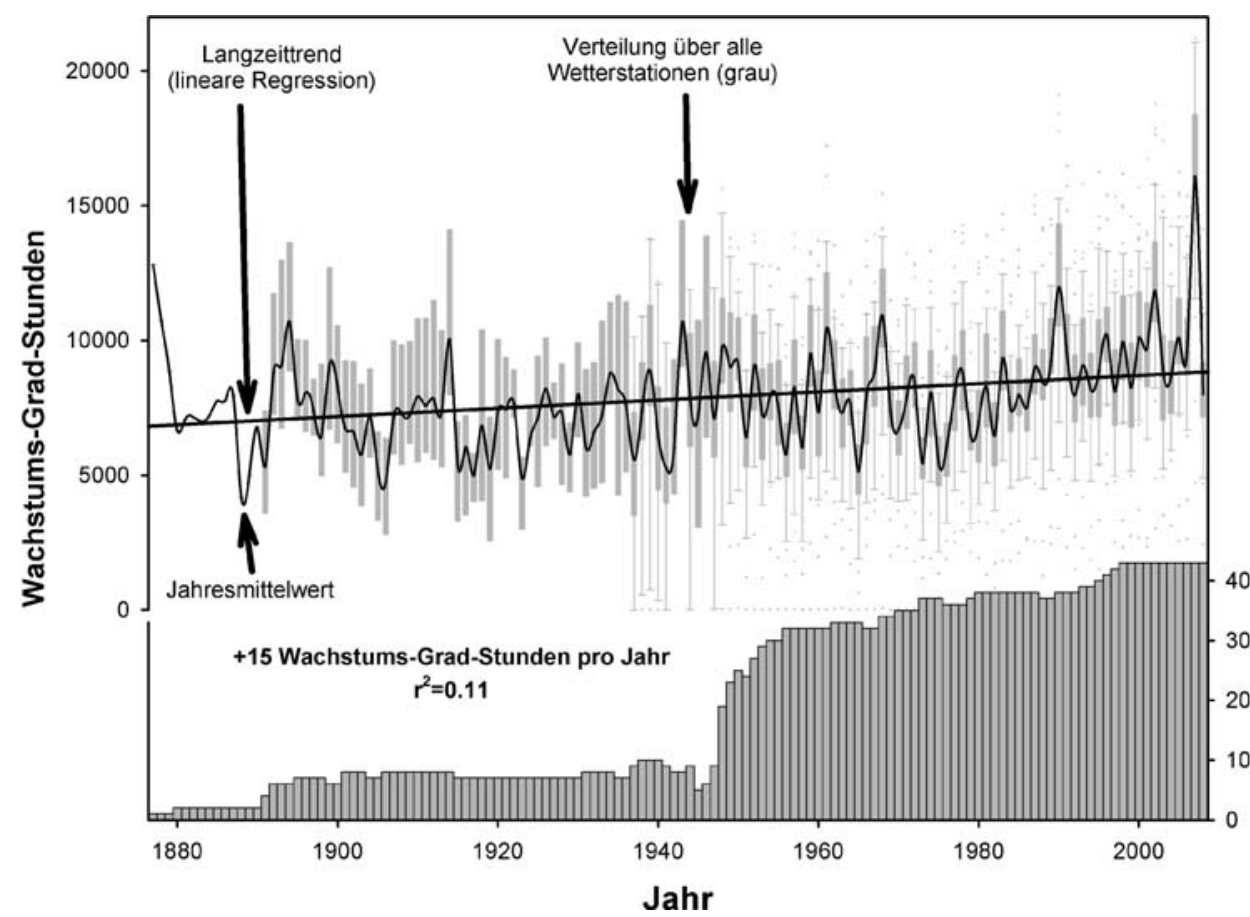

Frost) nach diesem Zeitpunkt keine bedeutenden Auswirkungen auf die Dormanzbrechung haben. Dass die hohen Januartemperaturen offenbar zu einer verfrühten Blüte geführt haben, ist ein Anhaltspunkt dafür, dass selbst in einem Jahr mit durchschnittlicher Winterkälte bereits im Januar die Kältebedürfnisse vieler Bäume erfüllt waren.

Der Winter von 2006/07 weist daher auf ein möglicherweise sehr viel größeres Problem hin. Nach der in Kalifornien üblichen Quantifizierung der winterlichen Wärme in Wachstums-Grad-Stunden (engl. Growing Degree Hours) stellt sich heraus, dass der besagte Winter aus „Obstbaumperspektive" der mit Abstand wärmste seit Beginn der Aufzeichnungen war (Abb. 10). Die Betrachtung dieses agroklimatischen Indikators ergibt einen deutlichen Erwärmungstrend über die vergangenen 130 Jahre.

Sollten die Kältebedürfnisse tatsächlich relativ früh im Winter erfüllt sein, dann hat das zur Folge, dass die Obstgehölze sehr viel früher genügend Wärme für die Blütenentwicklung ansammeln können und sich damit der Blühzeitpunkt verfrüht. In diesem Fall steigt die Wahrscheinlichkeit, dass die Blüte während einer relativ kalten Phase stattfindet. Die Folgen können negative Auswirkungen auf die BlühSynchronität und die Bestäubung und ein erhöhtes Risiko von Frostschäden sein.

\section{Schlussfolgerungen}

Für Deutschland insgesamt scheint die Kältewirkung während des letzten Jahrhunderts nicht oder nur sehr schwach abgenommen zu haben. Generell liegt sie zwischen etwa 1700 und 3000 Kältestunden oder zwischen 125 und 150 Kälteportionen, wobei die Regionen mit dem meisten Chilling eher im norddeutschen Raum als im kälteren Süden des Landes liegen. Das Kältestunden-Modell reagiert sehr viel empfindlicher auf Klimaänderungen als das KälteportionenModell. Aus dieser Erkenntnis ergibt sich die Fragestellung, welches der beiden Modelle, mit ihren sehr unterschiedlichen Ansätzen, für Deutschland am besten geeignet ist.

Es sieht nicht so aus, als würden dem deutschen Obstanbau auf absehbare Zeit durch Chilling-Verluste große Schwierigkeiten erwachsen. Ein größeres Problem könnte die Zunahme der winterlichen Wärme darstellen, die eine Verfrühung der Blütezeitpunkte und damit eine erhöhte Empfindlichkeit gegenüber Spätfrösten mit sich bringt.

Open Access Dieser Artikel wird zu den Bedingungen der "Creative Commons Attribution Noncommercial License" zur Verfügung gestellt. Damit ist eine nichtkommerzielle Nutzung, Verbreitung und Vervielfältigung erlaubt, sofern die Autoren des Artikels und die genaue Quelle angegeben sind.

\section{Literatur}

Anderson JL, Seeley SD (1992) Modelling strategies in pomology: development of the Utah models. Acta Hort 313:297-306

Baldocchi D, Wong S (2008) Accumulated winter chill is decreasing in the fruit growing regions of California. Clim Chang 87:S153-S166

Bennett JP (1949) Temperature and bud rest period. California Agric $3: 9+12$ 
Blanke M, Kunz A (2009) Einfluss rezenter Klimaveränderungen auf die Phänologie bei Kernobst am Standort Klein-Altendorf - anhand 50-jähriger Aufzeichnungen. Erwerbs-Obstbau 51 (in dieser Ausgabe) DOI 10.1007/s10341-009-0086-3

Byrne DH, Bacon TA (2009) Chilling accumulation: its importance and estimation. http://aggie-horticulture.tamu.edu/stonefruit/ chillacc.htm. Letzter Zugriff 4. März 2009

Chuine I, Cour P (1999) Climatic determinants of budburst seasonality in four temperate-zone tree species. New Phytologist 143:339_ 349

Deutscher Wetterdienst (2009) www.deutscherwetterdienst.de. Letzter Zugriff 1. Februar 2009

Erez A, Couvillon GA, Hendershott CH (1979a) Effect of cycle length on chilling negation by high-temperatures in dormant peach leaf buds. J Am Soc Hortic Sci 104:573-576

Erez A, Couvillon GA, Hendershott CH (1979b) Quantitative chilling enhancement and negation in peach buds by high-temperatures in a daily cycle. J Am Soc Hortic Sci 104:536-540

Fischer G, Lüdders P (1995) Der Apfelanbau im Hochland Kolumbiens. Erwerbsobstbau 37:58-62

Fishman S, Erez A, Couvillon GA (1987a) The temperature-dependence of dormancy breaking in plants - computer-simulation of processes studied under controlled temperatures. J Theor Biol 126:309-321

Fishman S, Erez A, Couvillon GA (1987b) The temperature dependence of dormancy breaking in plants: mathematical analysis of a two-step model involving a cooperative transition. J Theor Biol 124:473-483

Friedrich G, Fischer M (2000) Physiologische Grundlagen des Obstbaues. Verlag Eugen Ulmer, Stuttgart (Hohenheim)

Knight TA (1801) Account of some experiments on the ascent of the sap in trees. Philos Trans R Soc Lond 91:333-353

Linkosalo T, Hakkinen R, Hanninen H (2006) Models of the spring phenology of boreal and temperate trees: is there something missing? Tree Physiology 26:1165-1172

Linsley-Noakes GC, Allan P (1994) Comparison of 2 models for the prediction of rest completion in peaches. Sci Hort 59:107113
Linvill DE (1990) Calculating chilling hours and chill units from daily maximum and minimum temperature observations. Hort Sci 25:14-16

Luedeling E, Gebauer J, Buerkert A (2009a) Climate change effects on winter chill for tree crops with chilling requirements on the Arabian Peninsula. Clim Chang DOI:10.1007/s10584-10009-1958110587

Luedeling E, Zhang M, Luedeling V, Girvetz EH (2009b) Sensitivity of winter chill models for fruit and nut trees to climatic changes expected in California's Central Valley. Agric Ecosys Environ 133:23-31

Luedeling E, Zhang M, McGranahan G, Leslie C (2009c) Comparison of winter chill models for explaining walnut phenology. Agr Forest Meteorol (im Druck) DOI 10.1016/j.agrformet.2009.06.013

Luedeling E, Zhang M, Girvetz EH (2009d) Climatic changes lead to declining winter chill for fruit and nut trees in California during 1950-2099. PLoS ONE DOI 10.1371/journal.pone.0006166

Müller M, Braun P (2008) Development of phenological models over time - a review. Acta Hort 803:111-116

Richardson EA, Anderson JL, Campbell RH (1986) The omnidata biophenometer (TA45-P): a chill unit and growing degree hour accumulator. Acta Hort 184:95-100

Richardson EA, Seeley SD, Walker DR (1974) A model for estimating the completion of rest for redhaven and Elberta peach trees. Hortscience 9:331-332

Saure MC (1985) Dormancy release in deciduous fruit trees. Hortic Rev 7:239-300

Shaltout AD, Unrath CR (1983) Rest completion prediction model for Starkrimson Delicious apples. J Am Soc Hortic Sci 108:957-961

Vegis A (1961) Samenkeimung und vegetative Entwicklung der Knospen. Handbuch der Pflanzenphysiologie - Encyclopedia of Plant Physiology 16:168-298

Weinberger JH (1950) Chilling requirements of peach varieties. P Am Soc Hortic Sci 56:122-128

Westwood MN (1993) Temperate-zone pomology, 3rd edn. Timber Press, Portland, OR, USA

Winter F, Janssen H, Kennel W, Link H, Scherr F, Silbereisen R, Streif J (1992) Lucas' Anleitung zum Obstbau. Verlag Eugen Ulmer, Stuttgart 